\title{
Gestión del conocimiento para el desarrollo local de comunidades costeras en condiciones de fragilidad ambiental. Isabela de Sagua, Cuba.
}

\author{
Alonso Freyre, Joaquín \\ Universidad Central "Marta Abreu" de Las \\ Villas, Cuba \\ joaquin@uclv.edu.cu \\ Rodríguez Moya, Edelkis \\ Centro de Estudios y Servicios Ambientales \\ de Villa Clara, Cuba \\ director@cesam.vcl.cu
}

\author{
Garcés González, Roberto \\ Universidad Central "Marta Abreu" de Las \\ Villas, Cuba \\ rgarces@uclv.edu.cu
}

\section{Resumen}

El conocimiento desempeña un lugar esencial para el desarrollo en el mundo contemporáneo por su crecimiento exponencial y por su incidencia en la vida social. Su gestión ha devenido una cuestión de primer orden por la necesidad de propiciar su empleo en los más diversos procesos sociales, desde los más relevantes a nivel macro hasta los de vida cotidiana. En ello las universidades tienen un encargo que va más allá de la formación de profesionales al ser instituciones que lideran la producción, difusión y uso del conocimiento.

En Cuba la gestión el conocimiento es una práctica extendida como contribución a los procesos de desarrollo local comunitario, junto con la difusión de Centros Universitarios Municipales con capacidad para propiciar la continuidad del aprendizaje en todos los sectores de la sociedad y para la producción científica con alto rigor para la solución de problemáticas locales.
Ello no significa que los resultados que se observan en la gestión del conocimiento y en el aprovechamiento de las oportunidades que ofrece la presencia de la universidad en los municipios se hayan extendido a toda la geografía nacional como se muestra en el caso que se describe en una comunidad costera en condiciones de fragilidad ambiental.

Palabras clave: Gestión del conocimiento; diálogo de saberes; innovación; comunidades costeras; fragilidad ambiental;

\section{Abstract}

Knowledge plays an essential place for development in the contemporary world due to its exponential growth and its impact on social life. Its management has become a matter of the first order because of the need to promote its use in the most diverse social processes, from the most relevant at the macro level to those of daily life. In this the universities have an assignment that 
goes beyond the training of professionals to be institutions that lead the production, dissemination and use of knowledge.

In Cuba, knowledge management is an extended practice as a contribution to local community development processes, along with the dissemination of Municipal University Centers with the capacity to promote the continuity of learning in all sectors of society and for scientific production with high rigor for the solution of local problems.

This does not mean that the results observed in knowledge management and in taking advantage of the opportunities offered by the presence of the university in the municipalities have been extended to the entire national geography as shown in the case described in a coastal community in conditions of environmental fragility.

Keywords: Knowledge management; knowledge dialogue; innovation; coastal communities; environmental fragility;

\section{EL CONOCIMIENTO Y SU}

\section{GESTIÓN HOY}

El conocimiento es ahora parte sustancial del motor de acumulación por lo que en la actualidad se aprecia mejor su papel como factor condicionante del desarrollo. Es real que, a causa de las circunstancias creadas por el auge de las nuevas tecnologías, hoy constituye una variable decisiva en la productividad y en la competitividad de empresas y regiones a nivel internacional. (Castell, 2007). Eso también ocurre en la vida de las personas porque la tecnología interactúa con todos de modo transversal, atravesando la diversidad de actividades laborales, familiares, intelectuales, recreativas y las demás. (Martín Barbero, 1999).

El valor estratégico del conocimiento es innegable, porque está en constante expansión y como producto se revaloriza en la solución de las contradicciones y en los procesos regulares que ocurren en las organizaciones y en la vida social. El conocimiento, a diferencia del capital físico, aumenta de valor con su uso y pierde valor cuando no es utilizado.

Es un activo intangible que supera a otros productos y aporta un enorme valor agregado a los productos finales. Existe como aceptación generalizada, desde finales del siglo $X X$, el criterio de que la ventaja competitiva en las organizaciones, regiones y países está relacionada con la eficacia con que se realice el empleo intensivo del conocimiento. (Castell, 2007; Drucker 2006). Esta tendencia se con- 
vierte en regularidad internacional porque los saberes se crean y pierden novedad rápidamente en un mundo cada vez más globalizado donde los conocimientos y su gestión se han convertido en una de las principales cuestiones que reflejan la actualidad económica de los países más desarrollados que manifiestan así su necesidad de poder sirviéndose del conocimiento para mantener su hegemonía, porque el papel de este ha estado frecuentemente asociado al poder en los ejes de la dominación, de modo que es evidente la no neutralidad del conocimiento. (Foucault 1973)

\section{Según Habermas (2003:3);}

"La masa de la población trabajadora que desde hace siglos laboraba en el campo se desplaza primero al sector secundario, la industria productora de bienes, luego al terciario, el del comercio, el transporte y los servicios. Mientras tanto las sociedades postindustriales han desplegado un cuarto sector, el del conocimiento, que domina las industrias high-tec, los bancos o la administración pública, que dependen de la afluencia de nuevas informaciones y, en el último tiempo, de investigaciones $y$ avances en los sistemas de la informática".
Esta situación ha favorecido el surgimiento de una nueva tendencia para describir el nivel de desarrollo alcanzado en esta época donde la acumulación creciente de los conocimientos y su uso en la creación de nuevos productos y procesos constituye ahora uno de los pilares fundamentales de las denominadas "sociedades del conocimiento" (Bell, 1976; Castells, 2007).

El conocimiento en su crecimiento se comporta de manera exponencial, mientras que la capacidad cognitiva individual de apropiación de saberes sigue un paso aritmético. Esto provoca la ignorancia y la alienación de gran parte de la población que no es capaz de comprender cognitivamente la marcha del mundo actual. Sin embargo, en el mundo contemporáneo, el conocimiento pudiera ser "...fuente de desarrollo para la humanidad, sin exclusión de países y personas menos desarrollados" (Núñez Paula, 2007:2).

Esa es la esencia de la singularidad del caso de Cuba, que ha demostrado como país su capacidad para asegurar la continuidad del 
aprendizaje en todos los sectores de la sociedad, desde lo nacional hasta los municipios, porque a partir de la Campaña de Alfabetización en 1961 creó las bases de la igualdad de oportunidades para la libre y gratuita adquisición de los conocimientos y el acceso a todos los niveles de la Educación lo que hoy puede ayudar a la sociedad cubana a potenciar con éxito la apropiación social del conocimiento para emplearlo en los procesos de desarrollo local, teniendo en cuenta la necesidad de usar el conocimiento en una dirección acorde con valores predominantes en el país y que son junto a todo el potencial científico técnico creado por la Revolución Cubana parte indisoluble de la riqueza patrimonial del país y su apropiación es un bien al alcance de todos y para el disfrute pleno de la sociedad.

La apropiación social del conocimiento, en el caso de Cuba es un derecho del pueblo y un deber además para contribuir al desarrollo del país, conceptualmente es

“...el proceso mediante el cual las personas participan de actividades de producción, adaptación, aplicación de conocimientos y ac- ceden a sus beneficios. Significa que la gente interioriza el conocimiento y lo convierte en referente para el juicio y la actividad" (Núñez Jover, 2011).

El conocimiento como producto social es un recurso promisorio; pero solo no puede crear riquezas, también necesitade un sistema que lo gestione y de un entorno dinámico. (Bacallao, 2004; Castro DiazBalart, 2006 y Alhama, 2008). La literatura internacional ofrece diferentes perspectivas para solucionar ese problema. Entre esos esfuerzos aparece la gestión del conocimiento (GC), que surge en los años 90 del siglo $\mathrm{XX}$ y la novedad del concepto ha provocado mucho interés en el mundo académico y empresarial.

La gestión del conocimiento surgió legitimada por los intereses y las necesidades del empresariado capitalista contemporáneo y fue concebida para utilizar mejor las potencialidades del conocimiento en las organizaciones, permitiéndoles crecer en eficiencia y eficacia y obtener ventajas competitivas que aseguren la permanencia y dominio 
en el mercado. El gran reto es que el conocimiento propiamente dicho no se puede gestionar como tal. Sólo es posible gestionar el proceso y el espacio para la creación de conocimiento. (Osorio, 2003; Di Doménico, 2008; Davenport y Prusak, 1998).

El conocimiento no es un producto almacenado en un lugar junto a otros objetos manipulables; es una capacidad humana, es un proceso dinámico y lo relacionado con su gestión no es sobre él en sí mismo; sino encaminado a influir con objetivos determinados sobre las formas de identificarlo, adquirirlo, crearlo, desarrollarlo, compartirlo y conservarlo; es decir, la gestión está encaminada entonces a situarlo donde es pertinente y a promover su flujo; por lo tanto la GC se refiere a procesos sociales.

La función fundamental que se atribuye a la GC es articular el conocimiento con el problema a resolver. Está claro que el conocimiento por sí solo no conduce a mejores resultados, solo su gestión eficiente puede garantizar esta asociación entre él y las posibles soluciones y eso tiene que realizarse en un ambiente de integralidad que solo le puede dar el trabajo en colectivo, compartiendo proyectos, en redes de actores, en correspondencia con las opciones estratégicas en los contextos donde se ejecute.

En un municipio cubano la GC es una necesidad emergente, mucho más después de la aprobación de la nueva Constitución de la República que les transfiere grandes cuotas de autonomía y descentralización. Allí la articulación entre conocimientos relevantes y problemas tiene que transitar por procesos relacionados con la necesidad de identificar, adquirir, desarrollar, compartir, utilizar y retener el conocimiento que es necesario para fomentar los procesos de desarrollo local todo lo que requiere atención conceptual y práctica, porquelos problemas a abordar, como la alimentación, vivienda, salud, cuidado del medio ambiente, entre otros, son de carácter complejo y reclaman enfoques integradores interdisciplinarios, en los que las soluciones dependan de la promoción de acciones de GC que incentiven diálogos de saberes a través 
de la combinación racional de los conocimientos existentes, con otros adquiridos, fomentando la apropiación social del conocimiento y el bienestar humano local.

Estas ideas conducen a ponderar el papel de lo comunitario como cualidad en los procesos de desarrollo local, porque tanto la gestión del conocimiento como el desarrollo local son procesos sociales.

Una de las contradicciones más importantes que tienen ante sí los procesos de desarrollo local en $\mathrm{Cu}$ ba se refiere a que el país ha creado una enorme capacidad intelectual, que es una de sus principales riquezas potenciales, pero no la logra explotar eficientemente. Esta contradicción se hace más evidente en los escenarios locales.

El famoso escritor libanés Kalhil Gibrán, expresaba que: "Un poco de conocimiento operante vale infinitamente más que un gran caudal de saber inactivo" (Gibrán, 2007:50). Esta aseveración tiene una validez extraordinaria, porque en la sociología del conocimiento se insiste en el estudio de los orígenes sociales de las ideas y del efecto que las ideas dominantes tienen sobre las sociedades y profundiza en el tipo de relación del conocimiento con la sociedad. (Cores, 1975). En el caso concerniente al desarrollo local es mucho más válido, porque todos los saberes son válidos de acuerdo a cuáles sean las circunstancias que los demanden.

La GC en el municipio puede fortalecer la articulación entre actores sociales locales que operan en diferentes contextos, redimensionaa la conciencia crítica ya la creatividadcomo procesos socialescotidianos en la determinación y superación de las contradicciones, incentivar el diálogo de saberes sobre la base de la cooperación entre los actores implicados, promover la inclusión y la participación social, condicionando positivamente los procesos de innovación local que son elementos que fortalecen la condición humana y el espíritu comunitario como cualidad de los procesos de desarrollo.La GC no puede aplicarse de manera mecanicista, sino en correspondencia con 
las características de cada contexto y es importante atender a la calidad de la comunicación y el conjunto de relaciones que se crean donde se realiza.

En tal escenario la GC, como acción inmediata, se dirige a la coordinación de acciones encaminadas a aportar el tipo de conocimiento que puede contribuir a la solución de problemas en un lugar determinado y en el plano estratégico permite combinar prospectivamente el conocimiento con las proyecciones de desarrollo para contribuir a su materialización. De ahí que la GC puede ser definida como:

“...el conjunto de acciones, procesos, vínculos intersubjetivos que permiten que el patrimonio intelectual de una entidad o lugar como suma de capacidades y valores individuales, colectivos y locales se incremente de manera significativa en correspondencia con los recursos existentes y las ideas estratégicas que orientan sus procesos de desarrollo y está dirigida a identificar, adquirir, desarrollar, difundir, utilizar $y$ retener los conocimientos relevantes" (Garcés, 2013, p. 58)
En esta definición se reconoce que el conocimiento que existe en los municipios es la mayor riqueza que está al alcance de los líderes locales; pero la cantidad de resistencias que prevalecen en las rutinas organizacionales locales son una amenaza a tener en cuenta cuando se proyecta estratégicamente la GC y mucho más en contextos locales, que por múltiples causas pueden ser más conservadores porque los modos habituales de realizar las actividades están tan arraigados que es difícil cambiarlos.

Existen además determinadas resistencias asociadas a un grupo de tabúes que consideran a que las acciones de GC, por lo general no reflejan sus resultados en el corto plazo, criterios divergentes sobre si la GC es una inversión o un gasto, la tendencia de los empresarios trabajan más directamente vinculados a las inversiones en el corto plazo y las acciones a largo plazo son abordadas con mayor discreción, la orientación a ver ala GC totalmente relacionada con la formación del personal, ya sea desde la adquisición de los conocimientos hasta su difusión y la generalización de las 
mejores prácticas, la creencia de que los modelos de GC y los de Capital Intelectual de forma independiente, incluso de esa forma son estudiados y no se ha visto eldesarrollo del Capital Intelectual como el resultado de la GC y a pesar de que generalmente se reconoce el papel estratégico del conocimiento no siempre se usa de esa forma.

También existen otras limitaciones que pueden entorpecer la GC, ya en la propia marcha de los procesos de desarrollo local: que están relacionadas con las imprecisiones en los objetivos, la insuficiente planificación, la asignación difusa de responsabilidades, las confusiones conceptuales, la falta de una cultura adecuada, entre otras.

\section{Los Centros}

\section{UNIVERSITARIOS MUNICIPALES}

El recurso local más importante del municipio, como fruto de la política educacional del Estado cubano, es el conocimiento y su variedad en los diferentes contextos locales, sin embargo, esta oportunidad no ha sido suficientemente explotada en los procesos de desarrollo local que están en marcha en el país donde prevalece la costumbre de esperar por ideas preconcebidas.

También es preciso reconocer que hacia el interior del municipio existen diferentes localidades que poseen dinámicas propias, por tanto la municipalidad no debe reproducir en su relación con ellas los métodos de los que ha sido objeto en su relación de subordinación con la instancia provincial. Estas problemáticas reclaman atención, tanto de los que diseñan las políticas, como de los académicos que estudian el tema, en especial desde las ciencias sociales, porque es una contradicción que hace inoperante una de las principales oportunidades de usar el capital intelectual disponible para el municipio, y que está dentro de él, en función de catalizar con mayor pertinencia sus procesos de desarrollo local en una etapa en la que el país lucha por actualizar su modelo económico.

En el caso de Cuba, existe la posibilidad inédita en otros países de contar con la ventaja de tener en casi todos los municipios los Centros Universitarios Municipales 
(CUM), verdaderas forjas de capital humano local e interface segura entre el sistema de ciencia e innovación tecnológica del país y las localidades.El municipio, al contar con su existencia asegura la posibilidad de contar con un tipo de organización que flexibiliza el proceso local de gestión del conocimiento y asegura la posibilidad de obtener, con inmediatez, un vínculo con otros centros de Educación Superior y de investigaciones científicas del país, que a su vez tienen poderosos vínculos con redes internacionales del conocimiento. Todo ese flujo puede gravitar hacia el municipio y fertilizar sus procesos (Garcés, 2014).

El CUM es fundamental para el desarrollo del municipio. "Pero no cualquier CUM. Se necesitan CUM fortalecidos que actúen como agentes del conocimiento y la innovación. CUM que aglutinen agrónomos, ingenieros industriales, gente de ciencias sociales, econo- mistas, etc. Unos como plantilla y otros contratados..." (Núñez Jover, 2018, p.7).

EI CUM puede ser interface segura si actúa como el principal aliado del Gobierno y sirve a la vez de observatorio que haga vigilancia tecnológica para captar lo que el municipio necesite y demande en materia de saberes relevantes, y a la vez, es el centro articulador de todos los saberes locales, y aquí la palabra todos adquiere un verdadero sentido porque en el desarrollo del municipio son necesarios no solo los saberes académicos, sino todos los saberes que en un momento se pueden constituir en conocimientos relevantes. "...parte de la fuerza de los CUM radica en la articulación con las universidades de mayor porte y los centros de investigación y sus redes de trabajo. GUCID ${ }^{1}$ como red nacional impulsa esa articulación" (Núñez Jover, 2018: 8).

1. Red Gestión Universitaria del Conocimiento y la Innovación para el Desarrollo. (GUCID) Surgió como Programa Ramal del Ministerio de Educación Superior en 2006, dirigida por el Doctor en Ciencias Jorge Núñez Jover, en ese momento Director de Postgrado de la Universidad de La Habana y Coordinador de la Cátedra Ciencia Tecnología y Sociedad de ese Centro de Educación Superior (CES) de la república de Cuba. Ahora sigue funcionando como Red y articula a profesionales de todos los CES del país y a toda una diversidad de actores sociales. ( $\mathrm{N}$ de los $\mathrm{A}$.) 
El CUM puede fomentar su potencial crítico y combinatorio y jugar el papel de mediador $y$ articulador en el diálogo de saberes, que no se produce en un momento determinado solamente, sino que debe ser condición natural que acompañe como práctica cotidiana a los procesos de desarrollo local y a toda la gestión de gobierno que lo asegura y dirige. Esta rica práctica es propia de cada lugar y nace de sus condiciones, refleja la madurez de sus vínculos y en ellas son tan válidos los conocimientos relacionados, por ejemplo, con los tratamientos más modernos contra el cáncer, como el simple hecho de conseguir, por ejemplo, quien sabe herrar un buey o un caballo, reparar una red de pesca o saber cómo se comportan en determinado sitio del municipio las corrientes de agua en épocas de intensas lluvias o de inundaciones costeras, porque lo que hace relevante a un conocimiento es su contexto de demanda y aplicación.

\section{LA COMUNIDAD COSTERA DE} ISABELA DE SAGUA

Los estudios del Panel Intergubernamental para el estudio del Cambio Climático (IPCC, por sus siglas en inglés) han significado desde sus inicios la alta vulnerabilidad a los efectos de las alteraciones climáticas de los Pequeños Estados Insulares en Desarrollo (PEID) de todo el cinturón tropical, que incluye los más diversos archipiélagos del planeta, en especial los del Caribe y el Indopacífico (IPCC, 2013).

En contraste con los estados continentales, los PEIDs tienen un recurso básico sumamente limitado y vulnerable ante los efectos del cambio climático: la tierra emergida. Además, sufren en su gran mayoría, el impacto que genera el asentamiento de grandes poblaciones en sus litorales costeros y ribereños (UNEP, 2008).

Estos procesos de asentamiento humano han decursado inicialmente por la deforestación de grandes áreas para habilitar espacios constructivos que alberguen a poblaciones autóctonas y a otras flotantes y 
usualmente temporales. Estas se vinculan a servicios turísticos, pesqueros, náuticos 0 constructivos. Son también estacionales y se incrementan en períodos de primavera y verano. Por lo general hacen colapsar sistemas simples de servicios no concebidos para el arribo de miles de personas a la vez.

Cuba no resulta una excepción en este tipo de comportamiento humano. Los estudios de Peligro, Vulnerabilidad y Riesgo y del Macroproyecto de Zonas Costeras del Ministerio de ciencia, Tecnología y Medio ambiente (CITMA) han constatado que Cuba tiene un importante número de poblados costeros bajo alta vulnerabilidad, que pueden desaparecer bajo agua en las modelaciones de elevación del nivel medio del mar al 2050 y al 2100. De igual forma son muy vulnerables a la surgencia del mar e inundaciones costeras al paso de huracanes de diferentes categorías (Juanes y col., 2014).

Isabela de Sagua en específico, es una pequeña comunidad de la zona costera de Villa Clara en el municipio de Sagua la Grande que enfrenta situaciones ambientales extremas en los frágiles ecosistemas que la circundan y donde sus pobladores obtienen o cultivan sus principales insumos vitales. No difiere su situación ambiental de otros sitios similares, dado lo global de los impactos ambientales, que tienen por tanto una expresión a escala local.

La agricultura se ve cada vez más restringida por la penetración de la salinidad en los suelos cultivables, que paulatinamente van perdiendo condiciones para las plantaciones de viandas, frutas, hortalizas y van cediendo espacio a especies oportunistas y tolerantes a altos gradientes de estrés salino, que en el mejor de los casos pueden funcionar como atenuadores de la energía del mar y área de crías de juveniles de peces, crustáceos y aves, como es el caso particular de los manglares.

La pesca, tanto comercial como la de consumo local, que desde la década de los 90's por las urgencias alimentarias del país durante la crisis denominada "Período Especial" dejó de ser deportiva, expe- 
rimenta hoy el resultado de la captura intensiva al que ha sido sometido el stock pesquero por la vía de grandes redes de arrastre y la pesca submarina selectiva e intensiva.

Todas las estadísticas de capturas muestran en la actualidad francas tendencias al declive, en algunas especies ya sin presencia, lo cual indica valores muy bajos de sus poblaciones en el medio natural. Es un caso clásico de autofagia de un sector económico que sobre consume el recurso que lo sustenta por encima de su umbral de tolerancia (Baisre, 2004).

A esta situación se suma el impacto de la elevación de la temperatura de las aguas y la baja en los valores generales de salud de los arrecifes de coral, principal ecosistema submarino para la reproducción y crecimiento de especies marinas comerciales. Los manglares de la zona también están impactados y en dinámica constante por los azotes de eventos extremos y de la inestabilidad climática que afecta a toda la isla (Planos; Vega y Guevara, 2013).
En otro sentido, el paso devastador del huracán Irma en septiembre de 2017, impactó significativamente la infraestructura del poblado, generando derrumbes totales, parciales y destrucción de sistemas de servicio a la localidad. La altura de las aguas, en la casi totalidad del poblado, alcanzó 1.5 metros y generó destrucción significativa para declarar a Isabela como el sitio de mayor desastre en la provincia ante el paso de este evento meteorológico extremo (Delegación CITMA VC, 2017).

Se observa también el impacto progresivo que va teniendo la elevación del nivel medio del mar. Recurrentemente se inundan las zonas más bajas del poblado y esto provoca deterioro en las fuentes de abasto de agua, los canales de drenaje, los sembrados familiares e hidropónicos y los viales interiores. Las zonas más vulnerables se ubican hacia la ribera del rio Sagua la Grande, donde se combinan las escorrentías del rio y la entrada de la pleamar. 
El represamiento del Rio en un embalse algunos kilómetros aguas arriba como Alacranes, que no dispone de manejo de compuertas, ha ido generando también la penetración de la cuña salina rio arriba, dada la pérdida consecutiva de caudal ecológico suficiente. Especies de agua dulce que lo habitaban han visto muy disminuidas sus poblaciones y se van asentando otras tolerantes a medios salobres.

De igual forma, eventos deportivos que se realizaban en el rio, en especial el conocido como "Descenso del Rio Sagua", y que vinculaban vía fluvial las culturas de la capital municipal con Isabela, han desaparecido por la mala calidad de las aguas, contaminadas por múltiples fuentes emisoras de contaminación orgánica, inorgánica y de metales pesados. La pérdida de caudal del rio ha causado en ocasiones el afloramiento y concentración de grandes balsas de plantas acuáticas que bloquean la navegación a remos por el rio en grandes tramos. Los fuertes movimientos demográficos, la emigración de jóvenes y la escasez de ofertas laborales conspiran también contra la estabilidad de esta comunidad.
El punto de cambio negativo histórico más significativo en el declive de la economía de Isabela ocurrió con el cierre del puerto en la década del 90 , que constituía el ente económico fundamental de este poblado y el punto de intercambio mayor de que disponía la provincia con el exterior. El puerto involucraba trabajadores directos y de otros servicios alternativos que se conectaban al mismo y oxigenaba las finanzas, la comercialización interna de la localidad y su funcionamiento (Rodríguez y col., 2008).

Sin embargo, los habitantes de Isabela han recuperado en poco más de un año la funcionalidad local conectados participativamente para la recuperación del poblado, mostrando así capacidad de resiliencia, que denota actitudes claves para el trabajo comunitario integrado. Surgieron y se reafirmaron líderes populares en este Consejo Popular y la actuación de los pobladores fue positiva y de apoyo a la rehabilitación.

Los restaurantes particulares reabrieron sus puertas en poco más de un mes y los servicios estatales de igual forma, así como las instalaciones para la pesca. 


\section{LA ALTERNATIVA ECONÓMICA DE DESARROLLO LOCAL}

En el momento actual surge el turismo costero como una oportunidad para el rescate económico de este poblado. Ocurre este aspecto casi a nivel global, dando uso a subsistemas claves para el desarroIlo litoral (Barragán J. M. 2003). Derivación que transcurre de la producción a los servicios turísticos, especialmente en zonas que han visto limitados sus potencialidades de producción pero que mantienen valores paisajísticos de gran atractivo aún.

Este arribo del turismo como alternativa económica para la zona oeste de la provincia y en especial para la ruta Sagua-Isabela, ha estado preconcebido en ideas conceptuales desde hace cierto tiempo atrás por parte de la Dirección Provincial de Planificación Física.

Su diseño inicial está basado en el turismo masivo de sol y playa. Sin embargo, Isabela de Sagua como comunidad costera de pequeño tamaño y vulnerabilidad ambiental no puede asumir un turismo de esta magnitud. Su estructura urbana y espacio físico edificable es muy limitado.

Por demás y significativamente, la cayería que la circunda es un grupo de cayos frágiles, con una dinámica activa en su formación geológica aún y asimilando los efectos del cambio climático global. Los cayos fundamentales que pueden albergar intervenciones turísticas son Esquivel, El Cristo, Blanquizal, que ya ha perdido gran parte de su playa por elevación del mar, Bahía de Cádiz y Lanzanillo. Ninguno de ellos tieneáreas amplias para edificar construcciones de sistema de servicios y hospedaje de gran magnitud.

Todo este movimiento de desarrollo turístico se ha venido pensando sin la dimensión social como elemento definitorio del alcance y tipo de turismo que requiere la comunidad implementar. No han existido los encuentros necesarios para esta toma de decisiones. Los intercambios de saberes históricos sobre el funcionamiento de los componentes físico-geográfico y ecológico de la zona han sido prác- 
ticamente nulos, aunque se reconoce que el enfoque es positivo y en la búsqueda de alternativas laborales para un poblado litoral que hoy ofrece limitados escenarios de subsistencia a sus habitantes.

Han existido decisiones verticalistas en este sentido y la participación y consulta pública de la comunidad para las definiciones del qué, cómo y dónde construir no han sido participativas ni populares.

Lo que realmente necesita el país hoy es que en comunidades pequeñas se genere e implemente un tipo de turismo diferente, que vaya al uso de lo autóctono como el valor principal. No tienen condiciones para asimilar el estereotipo de turismo masivo que aplica en otros sitios, sin embargo, la idiosincrasia pesquera, la sensibilidad por la conservación de su terruño, sus valores culturales y su mar los hace únicos. Tienen el potencial para desplegar una amplia gama de ofertas comunitarias, basadas en el turismo de naturaleza, que puede marcar una gran diferencia en cuanto al tipo, impacto y beneficios a la comunidad de un turismo a es- cala comunitaria. Un turismo que busque el vínculo a los quehaceres cotidianos y no a formas preconcebidas de vender comunidades inexistentes en la vida real.

Estos enfoques, que surgen de la participación, potencian la idiosincrasia de los lugareños como el principal valor turístico a compartir y activan a su alrededor sistemas de servicios comunitarios que conllevan a más altos niveles de participación y colaboración ciudadana.

\section{GESTIÓN DEL CONOCIMIENTO}

PARA EL MANEJO COSTERO EN CONDICIONES DE FRAGILIDAD

\section{AMBIENTAL}

En lo expuesto hasta aquí se evidencia el tratamiento de dos temas que no se conectan discursivamente: 1-la GC incluyendo los centros universitarios municipales como oportunidad y 2-la alternativa de desarrollo local posible para la comunidad costera de Isabela de Sagua en condiciones de fragilidad ambiental. Sin embargo, la cuestión no es solo discursiva, sino resultante de una práctica cotidiana que constituye uno de los obstáculos 
que enfrenta esa comunidad para su desarrollo, por tanto, lo discursivo está expresando la realidad existente.

De una parte, es ciencia constituida para el mundo lo expuesto sobre gestión de conocimiento; para Cuba es además una práctica extendida como contribución a los procesos de desarrollo local comunitario (Garcés, 2014) en lo cual juegan un importante papel las universidades de modo directo $y$ a través de los CUM como lo demuestra la Red GUCID.

De otra parte, ¿cómo comprender que en tal escenario de oportunidades en el país para una adecuada GC haya ocurrido en Isabela la autofagia del sector económico de la pesca producto de la captura intensiva por la vía de grandes redes de arrastre y la pesca submarina selectiva e intensiva?; ¿Por qué no se resuelve la pérdida del caudal ecológico suficiente el Rio Sagua que impida la penetración de una cuña salina rio arriba? ¿Cómo es que continúa la mala calidad de sus aguas, conta- minadas por múltiples fuentes emisoras de contaminación orgánica, inorgánica y de metales pesados? ¿Qué alternativa se planteó desde la ciencia el cierre del puerto ocurrido en la década del 90 y como contribuye hoy a la llegada turismo como alternativa económica? Son muchos y variados los actores locales implicados en el surgimiento de esas problemáticas y que deben ser parte de su solución mediante una adecuada GC entre otros modos de gestionar el desarrollo local.

La GC in situ no se debe quedar en diagnosticar problemáticas, sino además fundamentar alternativas y propiciar el encuentro de los actores locales relacionados con cada una, no para decirles lo que deben hacer, sino para colocar sus resultados científicos en lenguaje comprensible y tener la apertura suficiente para incorporar el conocimiento de los demás. Al respecto existen experiencias en el mundo que plantean la necesidad del encuentro entre gobierno-academiacomunidad, (León, 2019) cada uno de los cuales incluye varios actores locales. 
Al respecto, resulta llamativa la reacción de la población de Isabela ante un estudio realizado sobre $\mathrm{Pe}$ ligro, Vulnerabilidades y Riesgos $(P \vee R)^{2}$. La identificación de esa problemática en esa comunidad costera partió de un diagnóstico ambiental y de los criterios de la población obtenidos mediante el intercambio directo con ella sobre las experiencias vividas y mediante resultados de encuestas anteriores. Su resultado se limitó a constatarla existencia de una baja percepción de riesgo, junto con desmotivación y desconocimiento sobre los estudios de PVR y que predomina el criterio de que esos estudios son parte de una estrategia para reubicar el poblado. (Santos, 2019)

Tal criterio constituye una muestra de resistencia de la comunidad a la práctica de reubicación de los asentamientos costeros en condiciones de PRV. Tal reubicación constituye una medida que se adopta en el país para elenfrentamiento al cambio climático conocida como "Tarea Vida" (CITMA, 2017). Hoy, luego de pasar por la experiencia de una catástrofe natural y trabajar por la recuperación de su localidad demostrando una resiliencia fundada enaltos niveles de arraigo y sentido de pertenencia, es comprensible que se estime por la población de que tales estudios se hacen para justificar su salida del lugar.

Ello evidencia un desencuentro entre el saber científico y el comunitario pues se colocan en lados opuestos frente a la problemática del cambio climático como efecto de una inadecuada GC que no propicia un diálogo. Corresponde a las autoridades del municipio su solución aprovechando para ello las fortalezas de que dispone, entre ellas la existencia del CUM.

En Sagua, municipio en que se encuentra Isabela, radica un CUM con amplia experiencia de trabajo que viene realizando, entre otros,

2. Estos estudios se establecieron por la Directiva Nro. 1 del 2010 del Presidente del Consejo de Defensa Nacional "Para la Reducción de Desastres". Por esa Directiva se encarga al CITMA la realización de estudios que permitan caracterizar la situación existente, la identificación y zonificación de áreas con escenarios propicios para ello, la estimación de la vulnerabilidad y el riesgo para cada tipo de peligro y proponer medidas a los planes de Reducción de Desastres a todos los niveles. 
eventos científicos sobre cambio climático en los que participan especialistas de nivel provincial y nacional. Ese CUM fue fundador de la Red GUCID y es el único de Villa Clara que por la cantidad de Doctores en Ciencia que forman parte de su claustro, tiene su propio Consejo Científico. Además, posee un grupo de profesionales bien formados $y$ con un sentido profundo de su compromiso con el desarrollo del territorio que puede devenir en actores sociales que se constituyan en el elemento que falta en esta articulación social entre todos los interesados en potenciar los procesos de desarrollo local comunitario del municipio Sagua la Grande y todas sus localidades.

Queda a ellos, como profundos conocedores de la realidad local fortalecer ese papel y a los demás, que son actores sociales externos y que llegan con distintos saberes, reconocer al CUM como la interface, como el aliado imprescindible para intervenir en el municipio y garantizar la sostenibilidad de los múltiples esfuerzos realizados.

\section{BIBLIOGRAFÍA}

Alhama Betamaric, R. (2008). Capital humano. Autorrealización y reconocimiento social. La Habana: Editorial de Ciencias Sociales.

Bacallao Sánchez, E. (2004). Una visión desde la competitividad y la gestión del conocimiento. Ciencia, Innovación y desarrollo. Revista de información científica y tecnológica. 9, (2)

Baisre, J A. La Pesca Marítima en Cuba. Editorial Científico-Técnica. 372pp.

Barragán J. M. (2003) Medio Ambiente y Desarrollo en áreas litorales: introducción a la Planificación y Gestión Integradas. Universidad de Cádiz. Servicio de Publicaciones,306 pp.

Bell, Daniel. (1976). TheComing of PostIndustrialSociety: A Venture in Social Forecasting. USA: Basic Books.

Castells, M. (2007). Globalización, tecnología, trabajo, empleo y empresa.http://www.lafactoriaweb.com/articulo s/castells7.htm

Castro Díaz-Balart, F. (2006). Ciencia, Tecnología e Innovación: desafíos e incertidumbres para el Sur. Ediciones Plaza. La Habana.

CITMA. (2017). Enfrentamiento al Cambio Climático en la República de Cuba: Tarea Vida. La Habana: CITMATEL.

Cores, L. A. (1975). Sociología del Conocimiento: Enciclopedia internacional de Ciencias Sociales. Aguedas. Madrid.

Davenport, T y Prusak, L. (Eds.). (1998). Working Knowledge. Boston: Harvard Business Scholl Press. 
Delegación CITMA VC, (2017). Evaluación de impacto ambiental de desastres provocados por el huracán IRMA en Villa Clara. 122 pp. Informe Digital.

Di Doménico, A. (2008). El conocimiento como recurso, una ventaja competitiva. Biblioteca Central Nacional de Mar del Plata. Argentina. http://www.bibio1.mdp.edu.ar/

Drucker, P. (2006). La administración; la organización basada en la información; la economía y la sociedad. Editorial Norma. Bogotá. Colombia.

http://www.scribd.com/.../Examinacion-yEvaluacion-de-Los-Aprendizajeshttp://dialnet.unirioja.es/servlet/articulo? codigo $=3399450$

Foucault, M. (1973). De la arqueología a la dinástica. In Michel Foucault. Estrategias de poder. Obras esenciales. Volumen II, Barcelona, Paidós, 1999, pp. 145-157.

Garcés, R. (2013). La gestión del conocimiento como contribución al desarrollo del municipio de Remedios. Tesis de Doctorado. Centro de Estudios Comunitarios. Universidad Central "Marta Abreu" de Las Villas. Santa Clara.

(2014). Las dimensiones de la gestión del conocimiento y los procesos de desarrollo local comunitario. Acta Universitaria, 24(1), 60-68. Disponible en http://www.actauniversitaria.ugto.mx/index.php/acta/article/view/509/html_25

Gibrán, K. (2007). La voz del maestro. http://www.librosgratisweb.com/libros/lavoz-del-maestro.html

Habermas, J. (2003). Conocimiento e interés. Ciencia y técnica como ideología, Ed. Tecnos, Madrid.http://www.usuarios.lycos.es/Cantemar/Conocimiento.html
IPCC, 2013: Climate Change 2013; The Physical Science Basis. Contribution of Working Groups to the Fifth Assesment Report of the Intergovermental Panel on Climate Change. [Stocker,T.F, D.Qin, G.K. Plattner, M, Tignor, Sk. Allen, J. Boschung, A. Nuels, Y. Xia, V.Bex, and P.M. Midgley (eds.)] Cambridge University Press, United Kingdom and New York, NY, USA, $135 \mathrm{pp}$.

Juanes, J.L y col. 2014. Informe de Proyecto. MACROPROYECTO: Escenarios de peligro y vulnerabilidad de la zona costera cubana, asociados al ascenso del nivel medio del mar para los años 2050 y 2100 . PROYECTO 6. Actualización y evaluación de la evolución de las playas para los años 2050 y 2100. 35pp.Perdomo, M. E. (2008). Estrategia de Educación Ambiental para el desarrollo sostenible en Comunidades Costeras. Tesis en opción al grado de Doctor en Ciencias Sociales. CESAM, CITMA, 131 pp.

León, J. (2019) "Innovación de los procesos de adaptación al cambio climático en el hábitat. La experiencia de la Universidad del BioBio, Chile". Seminario internacional Rol de las universidades en la innovación de los procesos de adaptación del hábitat al cambio climático. Santa Clara, UCLV.

Martín Barbero, J. (1999). Las transformaciones del mapa cultural. Ámbitos 2. Sevilla. http://www.ull.es/publicaciones/latina/ambitos/ambitos2/barbero.html

Núñez Jover, J. (2011). El conocimiento entre nosotros: reflexiones desde lo social. Temas, No. 65 / enero-marzo. La Habana, 94-104. 
(2018): Cinco tesis para dirigentes locales, Folletos gerenciales, Volumen XXII, enero-junio, MES, ISSN:

1726-5851, RNPS: 0488, pp. 109-121. Disponible en https://folletosgerenciales.mes.gob.cu/index.php/folletosgerenciales/index

Núñez Paula, I. (2007). Gestión Humana o de Personas en la construcción de las sociedades del conocimiento.ACIMED, Vol. 16. No. 3.http://bvs.sld.cu/revistas/aci/vol16_3_07/aci10907.html

Osorio Núñez, M. (2003). El capital intelectual en la gestión del conocimiento.ACIMED V.11

N.6http://scielo.sld.cu/scielo.php?script=sci_arttext\&pid=S102494352003000600008

Planos, E; Vega, R y A, Guevara, Editores, (2013). Impacto del Cambio Climático y Medidas de Adaptación en Cuba. INSMET, AMA, CITMA, Cuba 430 pp.

Rodríguez, E y col. 2008. Desarrollo comunitario en Isabela de Sagua. Informe de Proyecto Territorial 0640. CESAM, CITMA, 27pp.

Santos Romero, Y. (2019) Implementación de los estudios de Peligro, vulnerabilidad y riesgo (PVR) con participación popular en la comunidad costera de Isabela de Sagua. Taller de tesis, Maestría en Desarrollo Comunitario. Santa Clara, UCLV.

UNEP (2008). Climate Change in the Caribbean and the Challenge of Adaptation.UNEP Regional Office for Latin American and the Caribbean, Panama City. 